\title{
WEIGHTED NORM INEQUALITIES FOR CERTAIN INTEGRAL OPERATORS. II
}

\author{
H. P. HEINIG ${ }^{1}$
}

\begin{abstract}
Conditions on nonnegative weight functions $u(x)$ and $v(x)$ are given which ensure that an inequality of the form $\left(\int|T f(x)|^{q} u(x) d x\right)^{1 / q} \leqslant$ $C\left(\int|f(x)|^{p} v(x) d x\right)^{1 / p}$ holds for $1 \leqslant q<p<\infty$, where $T$ is an integral operator of the form $\int_{-\infty}^{x} K(x, y) f(y) d y$ or $\int_{x}^{\infty} K(y, x) f(y) d y$ and $C$ a constant independent of $f$. Specifically a number of inequalities for well-known classical operators are obtained. Inequalities of the above form for $1 \leqslant p \leqslant q<\infty$ were obtained in [1].
\end{abstract}

1. Introduction. Let $K(x, y) \geqslant 0$ be defined on $\Delta=\left\{(x, y) \in \mathbf{R}^{2}: y<x\right\}$ and define $K$ and $K^{*}$ by

$$
(K f)(x)=\int_{-\infty}^{x} K(x, y) f(y) d y, \quad\left(K^{*} f\right)(x)=\int_{x}^{\infty} K(y, x) f(y) d y .
$$

In [1] conditions on nonnegative weight functions $u(x)$ and $v(x)$ were given, which imply norm inequalities of the form

$$
\left(\int_{-\infty}^{\infty}|(T f)(x)|^{q} u(x) d x\right)^{1 / q} \leqslant C\left(\int_{-\infty}^{\infty}|f(x)|^{p} v(x) d x\right)^{1 / p}
$$

for $1 \leqslant p \leqslant q<\infty$, where $T$ is either $K$ or $K^{*}$ and $C>0$ a constant independent of $f$.

The purpose of this paper is to extend the results of [1] in the sense that under similar conditions on $u$ and $v$ the norm inequality (1.2) holds for $1 \leqslant q<p<\infty$. If $q=1$, the conditions are also shown to be necessary. As in [1] the operators considered here include several classical operators such as the Riemann-Liouville and Weyl fractional integral operators, the Laplace transform, certain convolution operators, as well as others. In fact the class of operators considered here is somewhat larger than that considered in [1]. The results yield new integral inequalities and, as the estimate for the fractional integral operator shows, provide examples of translation invariant operators which map weighted $L^{p}$-spaces to weighted $L^{q}$-spaces for $q<p$. Further, the discrete analogues of the integral operators are discussed which extend the corresponding results of [1].

If $K(x, y) \equiv 1$ and $f$ is supported in $(0, \infty),(1.1)$ reduces to the Hardy operators, which were studied by Mazja [4] and Sawyer [3].

Received by the editors October 9, 1984.

1980 Mathematics Subject Classification. Primary 26D15; Secondary 26A33.

${ }^{1}$ Research supported in part by the Natural Sciences and Engineering Research Council Grant A4837. 
The plan of the paper is as follows: The next section contains the main results (Theorems 2.1 and 2.2) and some corollaries which illustrate the theorems for specific operators. In $\S 3$ the discrete case is briefly discussed.

Throughout, $p^{\prime}$ denotes the conjugate index of $p, p \geqslant 1$, defined by $p+p^{\prime}=p p^{\prime}$ and $p^{\prime}=\infty$ if $p=1$. The conjugate of $q$ is defined in the same way. Products of the form $0 \cdot \infty$ are taken to be zero and $A, B, C$ denote constants which may be different at different occurrences while $\mathbf{Z}$ denotes the set of integers.

It is a pleasure to thank Professor A. Kufner who drew my attention to Mazja's work and Professor E. T. Sawyer for providing a variant of Mazja's proof of the Hardy inequality.

2. Main results. The weight conditions we need are now given in the following definitions:

Definition 2.1. Let $u(x) \geqslant 0, v(x) \geqslant 0,1 \leqslant q<p<\infty$, and suppose $K(x, y)$ $\geqslant 0$ is defined in $\Delta=\left\{(x, y) \in \mathbf{R}^{2}: y<x\right\}$.

(a) We write $(u, v) \in B(K, p, q)$ if

$$
\begin{aligned}
B & \equiv\left(\int_{-\infty}^{\infty}\left[\left(\int_{y}^{\infty} K(x, y)^{q} u(x) d x\right)^{1 / q}\left(\int_{-\infty}^{y} v(x)^{1-p^{\prime}} d x\right)^{1 / q^{\prime}}\right]^{r} v(y)^{1-p^{\prime}} d y\right)^{1 / r} \\
& <\infty
\end{aligned}
$$

where $1 / r=1 / q-1 / p$. In case $q=1<p$, the condition takes the form

$$
B \equiv\left(\int_{-\infty}^{\infty}\left(\int_{y}^{\infty} K(x, y) u(x) d x\right)^{p^{\prime}} v(y)^{1-p^{\prime}} d y\right)^{1 / p^{\prime}}<\infty .
$$

(b) We write $(u, v) \in B^{*}(K, p, q)$ if

$$
\begin{aligned}
B^{*} & \equiv\left(\int_{-\infty}^{\infty}\left[\left(\int_{-\infty}^{y} K(x, y)^{q} u(x) d x\right)^{1 / q}\left(\int_{y}^{\infty} v(x)^{1-p^{\prime}} d x\right)^{1 / q^{\prime}}\right]^{r} v(y)^{1-p^{\prime}} d y\right)^{1 / r} \\
& <\infty
\end{aligned}
$$

where $1 / r=1 / q-1 / p$. If $q=1<p$ the condition is

$$
B^{*} \equiv\left(\int_{-\infty}^{\infty}\left(\int_{-\infty}^{y} K(x, y) u(x) d x\right)^{p^{\prime}} v(y)^{1-p^{\prime}} d y\right)^{1 / p^{\prime}}<\infty .
$$

Note that in the limiting case $q=p$ the integral in (a) takes the form

$$
\sup _{y}\left(\int_{y}^{\infty} K(x, y)^{p} u(x) d x\right)^{1 / p}\left(\int_{-\infty}^{y} v(x)^{1-p^{\prime}} d x\right)^{1 / p^{\prime}} \equiv B<\infty
$$

and similarly the integral in (b).

We now give the main results.

THEOREM 2.1. Let $K$ be the integral operator defined by $(1.1)$, where $K(x, y) \geqslant 0$ is defined in $\Delta$ and is nondecreasing in $y$. If $(u, v) \in B(K, p, q), 1 \leqslant q<p<\infty$, then

$$
\left(\int_{-\infty}^{\infty}|(K f)(x)|^{q} u(x) d x\right)^{1 / q} \leqslant B C\left(\int_{-\infty}^{\infty}|f(x)|^{p} v(x) d x\right)^{1 / p},
$$

where $C=q^{1 / p}\left(p^{\prime}\right)^{1 / q^{\prime}}$. 
WEIGHTED NORM INEQUALITIES

389

Moreover, if $q=1$, then $B(K, p, q)$ is necessary for (2.1).

Proof. Without loss of generality assume $f(x) \geqslant 0$ for which the right side of (2.1) is finite.

Let $q>1$ and set $\sigma(x)=v(x)^{1-p^{\prime}}$. Integration and an interchange of order of integration shows that

$$
\begin{aligned}
\int_{-\infty}^{\infty} & u(x)\left(\int_{-\infty}^{x} K(x, y) \sigma(y) f(y) d y\right)^{q} d x \\
& =q \int_{-\infty}^{\infty} u(x) \int_{-\infty}^{x}\left(\int_{-\infty}^{y} K(x, t) \sigma(t) f(t) d t\right)^{q-1} K(x, y) \sigma(y) f(y) d y d x \\
& =q \int_{-\infty}^{\infty} \sigma(y) f(y) \int_{y}^{\infty} u(x) K(x, y)\left(\int_{-\infty}^{y} K(x, t) \sigma(t) f(t) d t\right)^{q-1} d x d y \\
& \leqslant q \int_{-\infty}^{\infty} \sigma(y) f(y)\left(\int_{-\infty}^{y} \sigma(t) f(t) d t\right)^{q-1}\left(\int_{y}^{\infty} u(x) K(x, y)^{q} d x\right) d y,
\end{aligned}
$$

since $t<y<x$ implies $K(x, t) \leqslant K(x, y)$. Now writing

$$
\sigma(x)=\sigma(x)^{(q-1) / p+(p-q) / p+1 / p}
$$

and applying Hölder's inequality with indices $p, p /(q-1), p /(p-q)$ the last expression is not larger than

$$
\begin{aligned}
\left(\int_{-\infty}^{\infty} \sigma(y) f(y)^{p} d y\right)^{1 / p} \\
\cdot\left(\int_{-\infty}^{\infty}\left[\int_{-\infty}^{y} \sigma(t) f(t) d t / \int_{-\infty}^{y} \sigma(t) d t\right]^{p} \sigma(y) d y\right)^{(q-1) / p} \\
\cdot\left(\int_{-\infty}^{\infty}\left[\left(\int_{y}^{\infty} u(x) K(x, y)^{q} d x\right)^{1 / q}\left(\int_{-\infty}^{y} \sigma(t) d t\right)^{1 / q^{\prime}}\right]^{r} \sigma(y) d y\right)^{(p-q) / p} .
\end{aligned}
$$

The last integral of (2.2) is clearly $B^{q}$ (Definition 2.1 ), while the second integral is by [1, Theorem 2.1] with $K \equiv 1$ and $q=p$ not larger than

$$
(A C)^{q-1}\left(\int_{-\infty}^{\infty} \sigma(y) f(y)^{p} d y\right)^{(q-1) / p},
$$

where $A=\left(p^{\prime}\right)^{1 / p^{\prime}} p^{1 / p}$ and

$$
\begin{aligned}
C= & \sup _{a \in \mathbf{R}}\left(\int_{a}^{\infty}\left(\int_{-\infty}^{y} \sigma(t) d t\right)^{-p} \sigma(y) d y\right)^{1 / p}\left(\int_{-\infty}^{a} \sigma(t) d t\right)^{1 / p^{\prime}} \\
= & (p-1)^{-1 / p} \sup _{a \in \mathbf{R}}\left[\left(\int_{-\infty}^{a} \sigma(t) d t\right)^{1-p}-\left(\int_{-\infty}^{\infty} \sigma(t) d t\right)^{1-p}\right]^{1 / p} \\
& \cdot\left(\int_{-\infty}^{a} \sigma(t) d t\right)^{1 / p^{\prime}} \\
\leqslant & (p-1)^{-1 / p} .
\end{aligned}
$$


Therefore,

$$
\begin{gathered}
\left(\int_{-\infty}^{\infty} u(x)\left(\int_{-\infty}^{x} K(x, y) \sigma(y) f(y) d y\right)^{q} d x\right)^{1 / q} \\
\leqslant B q^{1 / q}\left(p^{\prime}\right)^{1 / q^{\prime}}\left(\int_{-\infty}^{\infty} \sigma(y) f(y)^{p} d y\right)^{1 / p}
\end{gathered}
$$

which is equivalent to (2.1).

If $q=1$, an interchange of order of integration and Hölder's inequality shows that

$$
\begin{aligned}
\int_{-\infty}^{\infty} & u(x)\left(\int_{-\infty}^{x} K(x, y) f(y) d y\right) d x \\
& =\int_{-\infty}^{\infty} f(y) v(y)^{1 / p-1 / p} \int_{y}^{\infty} K(x, y) u(x) d x d y \\
& \leqslant\left(\int_{-\infty}^{\infty} f(y)^{p} v(y) d y\right)^{1 / p}\left(\int_{-\infty}^{\infty} v(y)^{1-p^{\prime}}\left(\int_{y}^{\infty} K(x, y) u(x) d x\right)^{p^{\prime}} d y\right)^{1 / p^{\prime}} \\
& =B\left(\int_{-\infty}^{\infty} f(y)^{p} v(y) d y\right)^{1 / p}
\end{aligned}
$$

which is (2.1).

Conversely, if (2.1) holds with $q=1$, define $f$ by

$$
f(y)=v(y)^{1-p^{\prime}}\left(\int_{y}^{\infty} K(x, y) u(x) d x\right)^{1 /(p-1)}, \quad y \in \mathbf{R} .
$$

But since, after interchanging the order of integration, (2.1) with $q=1$ takes the form

$$
\int_{-\infty}^{\infty} f(y) \int_{y}^{\infty} K(x, y) u(x) d x d y \leqslant C\left(\int_{-\infty}^{\infty} f(y)^{p} v(y) d y\right)^{1 / p}
$$

we obtain

$$
\begin{aligned}
& \int_{-\infty}^{\infty} v(y)^{1-p^{\prime}}\left(\int_{y}^{\infty} K(x, y) u(x) d x\right)^{1+1 /(p-1)} d y \\
& \quad \leqslant C\left(\int_{-\infty}^{\infty} v(y)^{1-p^{\prime}}\left(\int_{y}^{\infty} K(x, y) u(x) d x\right)^{p^{\prime}} d y\right)^{1 / p}
\end{aligned}
$$

or

$$
\left(\int_{-\infty}^{\infty} v(y)^{1-p^{\prime}}\left(\int_{y}^{\infty} K(x, y) u(x) d x\right)^{p^{\prime}} d y\right)^{1 / p^{\prime}} \leqslant C .
$$

This completes the proof of the theorem.

Note that the proof of the theorem in the case $q=1$ does not require that $K(x, y)$ is nondecreasing in $y$. 
THEOREM 2.2. Let $K^{*}$ be defined by (1.1), where $K(x, y) \geqslant 0$ is defined in $\Delta$ and is nonincreasing in $x$. If $(u, v) \in B^{*}(K, p, q), 1 \leqslant q<p<\infty$, then

$$
\left(\int_{-\infty}^{\infty}\left|\left(K^{*} f\right)(x)\right|^{q} u(x) d x\right)^{1 / q} \leqslant B^{*} C\left(\int_{-\infty}^{\infty}|f(x)|^{p} v(x) d x\right)^{1 / p}
$$

where $C=q^{1 / q}\left(p^{\prime}\right)^{1 / q^{\prime}}$. Moreover, if $q=1$ then $B^{*}(K, p, q)$ is necessary for (2.3).

The proof of this result is almost identical to that of Theorem 2.1 , only now we apply [1, Theorem 2.2], and in the case $q=1$ one defines $f$ by

$$
f(y)=v(y)^{1-p^{\prime}}\left(\int_{-\infty}^{y} K(y, x) u(x) d x\right)^{1 /(p-1)}
$$

to show that (2.3) implies $B^{*}(K, p, 1)$. The details are omitted.

Corollary 2.1. Suppose $k(x) \geqslant 0$ is nonincreasing and $1 \leqslant q<p<\infty$. If $1 / r=1 / q-1 / p$ and

$$
\begin{array}{r}
B \equiv\left(\int_{-\infty}^{\infty}\left[\left(\int_{y}^{\infty} k(x-y)^{q} u(x) d x\right)^{1 / q}\left(\int_{-\infty}^{y} v(x)^{1-p^{\prime}} d x\right)^{1 / q^{\prime}}\right]^{r}\right. \\
\left.\cdot v(x)^{1-p^{\prime}} d x\right)^{1 / r}<\infty,
\end{array}
$$

( with the obvious modification if $q=1$ ), then

$$
\begin{gathered}
\left(\int_{-\infty}^{\infty} u(x)\left|\int_{-\infty}^{x} k(x-y) f(y) d y\right|^{q} d x\right)^{1 / q} \\
\leqslant B q^{1 / q}\left(p^{\prime}\right)^{1 / q^{\prime}}\left(\int_{-\infty}^{\infty} f(x)^{p} v(x) d x\right)^{1 / p} .
\end{gathered}
$$

A similar result holds for the dual operator.

If $k \equiv 1$ and $f$ is supported on $(0, \infty)$ one obtains the Hardy operator, and Corollary 2.1 yields the generalizations of Hardy's inequality given by Mazja [4] and independently by Sawyer [5] where even the case $0<q<p, p \geqslant 1$ was considered. Moreover, in this case (2.5) implies (2.4) and, similarly, for the dual operator.

If $k(x) \equiv x^{\alpha-1} / \Gamma(\alpha), 0<\alpha<1$, and $f$ is supposed on $(0, \infty)$, one obtains the Riemann-Liouville fractional integral operator

$$
\left(I_{\alpha} f\right)(x)=\frac{1}{\Gamma(\alpha)} \int_{0}^{x}(x-t)^{\alpha-1} f(t) d t .
$$

Corollary 2.1 then shows that

$$
\int_{0}^{\infty}\left[\left(\int_{y}^{\infty}(x-y)^{(\alpha-1) q} u(x) d x\right)^{1 / q}\left(\int_{0}^{y} v(x)^{1-p^{\prime}} d x\right)^{1 / q^{\prime}}\right]^{\prime} v(y)^{1-p^{\prime}} d y<\infty
$$

implies

$$
\left(\int_{0}^{\infty}\left|\left(I_{\alpha} f\right)(x)\right|^{q} u(x) d x\right)^{1 / q} \leqslant C\left(\int_{0}^{\infty}|f(x)|^{p} v(x) d x\right)^{1 / p},
$$

$1 \leqslant q<p<\infty$. A similar result holds for the Weyl fractional integral operator. 
Note that unlike the case $1 \leqslant p \leqslant q<\infty$ considered in [1], the condition on the weights in the case $q<p$ is more restrictive. In particular, if $u$ and $v$ are both power weights (2.6) cannot hold. However, for large classes of weights one does have weighted estimates. We single out the weights $u(x)=x^{a}$ and $v(x)=e^{x}$.

Corollary 2.2. If $1<q<p<\infty$ and $(1-\alpha) q-2+q / p<a<(1-\alpha) q-$ $1<0$, then (2.7) holds with $u(x)=x^{a}$ and $v(x)=e^{x}$.

Proof. We must show that the integral of (2.6) is finite. Since

$$
\int_{0}^{y} e^{x\left(1-p^{\prime}\right)} d x \leqslant 1 /\left(p^{\prime}-1\right)
$$

and

$$
\int_{y}^{\infty}(x-y)^{(\alpha-1) q} x^{a} d x=y^{(\alpha-1) q+a+1} B((\alpha-1) q+1,(1-\alpha) q-1-a),
$$

where $B$ is the Beta function and $(\alpha-1) q+1>0,(1-\alpha) q-1-a>0$, we obtain on substituting that the left side of $(2.6)$ is dominated by

$$
\int_{0}^{\infty} y^{r[(\alpha-1) q+a+1] / q} e^{-y\left(p^{\prime}-1\right)} d y .
$$

But this integral is finite if $r[(\alpha+1) q+a+1] / q+1>0$. Hence (2.6) holds if $(\alpha-1) q+a+1>-q / r$ and $0>(1-\alpha) q-1>a$. Since $1 / r=1 / q-1 / p$, (2.7) holds if $0>(1-\alpha) q-1>a>(1-\alpha) q-2+q / p$.

Observe also that

$$
\int_{0}^{x}(x-y)^{\alpha-1}|f(y)| d y \geqslant h^{\alpha-1} \int_{x-h}^{x}|f(y)| d y, \quad 0<h<x,
$$

so that $\left(M_{\alpha} f\right)(x) \leqslant \Gamma(\alpha)\left(I_{\alpha}|f|\right)(x)$ where

$$
\left(M_{\alpha} f\right)(x)=\sup _{0<h<x} h^{\alpha-1} \int_{x-h}^{x}|f(y)| d y
$$

is the left fractional maximal function. Therefore, the norm inequality for $I_{\alpha}$ implies the inequality for $M_{\alpha}$.

Corollary 2.3. Suppose $1 \leqslant q<p<\infty, 1 / r=1 / q-1 / p$.

(a) If $0<\gamma<1$ and

$$
\int_{-\infty}^{\infty}\left[\left(\int_{y}^{y / \gamma} u(x) d x\right)^{1 / q}\left(\int_{-\infty}^{y} v(x)^{1-p^{\prime}} d x\right)^{1 / q^{\prime}}\right]^{r} v(y)^{1-p^{\prime}} d y<\infty,
$$

then

$$
\left(\int_{-\infty}^{\infty} u(x)\left|\int_{\gamma x}^{x} f(y) d y\right|^{q} d x\right)^{1 / q} \leqslant C\left(\int_{-\infty}^{\infty}|f(x)|^{p} v(x) d x\right)^{1 / p} .
$$

(b) If $\gamma>0$ and

$$
\int_{-\infty}^{\infty}\left[\left(\int_{-\infty}^{y} e^{-\gamma q(x-y)} u(x) d x\right)^{1 / q}\left(\int_{y}^{\infty} v(x)^{1-p^{\prime}} d x\right)^{1 / q^{\prime}}\right]^{r} v(y)^{1-p^{\prime}} d y<\infty
$$

then

$$
\left(\int_{-\infty}^{\infty} u(x)|F(x)|^{q} d x\right)^{1 / q} \leqslant C\left(\int_{-\infty}^{\infty}|f(x)|^{p} v(x) d x\right)^{1 / p}
$$

where $F(x)=e^{\gamma x} \int_{x}^{\infty} e^{-\gamma t} f(t) d t$. 
Proof. (a) Define $K$ on $\Delta$ by $K(x, y)=1$ if $\gamma x<y$, and zero otherwise. Clearly $K$ is nonincreasing in $y$ and the result follows from Theorem 2.1.

(b) Let $K(x, y)=e^{-\gamma(y-x)}, y \geqslant x$, then $K$ is defined in $\Delta$ and nonincreasing in the first variable and (1.1) shows that $F(x)=\left(K^{*} f\right)(x)$. Therefore, the result follows from Theorem 2.2 .

Unweighted $L^{p}$-estimates of $F(x)$ were obtained by Stepanov (cf. [3, p. 26]).

The last application involves the Laplace transform $L$ defined by

$$
(L f)(x)=\int_{0}^{\infty} e^{-x t} f(t) d t, \quad x>0 .
$$

If $(K f)(x)=\int_{0}^{x} f(y) d y$ and $\left(K^{*} f\right)(x)=\int_{x}^{\infty} e^{-y / x} f(y) d y$, then, for $f \geqslant 0$,

$$
e^{-1}(K f)(1 / x)+\left(K^{*} f\right)(1 / x) \leqslant(L f)(1 / x) \leqslant(K f)(1 / x)+\left(K^{*} f\right)(1 / x),
$$

so that

$$
\left(\int_{0}^{\infty} u(x)[(L f)(x)]^{q} d x\right)^{1 / q} \leqslant C\left(\int_{0}^{\infty}[f(x)]^{p} v(x) d x\right)^{1 / p}
$$

if and only if

$$
\left(\int_{0}^{\infty} u(1 / x)(K f)(x)^{q} x^{-2} d x\right)^{1 / q} \text { and }\left(\int_{0}^{\infty} u(1 / x)\left(K^{*} f\right)(x)^{q} x^{-2} d x\right)^{1 / q}
$$

are bounded by a multiple of $\left(\int_{0}^{\infty} f(x)^{p} v(x) d x\right)^{1 / p}$. Now applying Theorems 2.1 and 2.2 we obtain at once the following result (cf. [1, Theorem 2.3] where the case $1 \leqslant p \leqslant q<\infty$ was considered):

THEOREM 2.3. If

$$
\int_{0}^{\infty}\left[\left(\int_{0}^{1 / y} u(x) d x\right)^{1 / q}\left(\int_{0}^{y} v(x)^{1-p^{\prime}} d x\right)^{1 / q^{\prime}}\right]^{r} v(y)^{1-p^{\prime}} d y<\infty
$$

and

$$
\int_{0}^{\infty}\left[\left(\int_{1 / y}^{\infty} e^{-x y q} u(x) d x\right)^{1 / q}\left(\int_{y}^{\infty} v(x)^{1-p^{\prime}} d x\right)^{1 / q^{\prime}}\right]^{r} v(y)^{1-p^{\prime}} d y<\infty,
$$

$1 \leqslant q<p<\infty, 1 / r=1 / q-1 / p$, then (2.8) holds.

If $1<q<p<\infty$ and $q / p<a<1$, then as a special case of Theorem 2.3 one obtains

$$
\left(\int_{0}^{\infty} x^{-a}|(L f)(x)|^{q} d x\right)^{1 / q} \leqslant C\left(\int_{0}^{\infty}\left|\left(1+x^{2}\right) f(x)\right|^{p} \frac{d x}{1+x^{2}}\right)^{1 / p} .
$$

To see this, let $u(x)=x^{-a}$ and $v(x)=\left(1+x^{2}\right)^{p-1}$ in the above integrals. Since $\left(1-p^{\prime}\right)(p-1)=-1$ and $-a+1>0$, the first integral is dominated by $\int_{0}^{\infty} y^{(a-1) r / q}\left(1+y^{2}\right)^{-1} d y$. But this integral is finite if $-1<(a-1) r / q<1$, that is $1-q / r<a<1+q / r$ but $1 / r=1 / q-1 / p$, so in particular if $q / p<a<1$. 
Similarly, the second integral in Theorem 2.3 is dominated by

$$
\begin{aligned}
\int_{0}^{\infty} & \left(1+y^{2}\right)^{-1}\left(\int_{1 / y}^{\infty} e^{-x y q} x^{-a} d x\right)^{r / q} d y \\
& =C\left(\int_{0}^{\infty} y^{(a-1) r / q}\left(1+y^{2}\right)^{-1} d y\right)\left(\int_{q}^{\infty} e^{-t} t^{-a} d t\right)^{r / q}, \quad t=x y q .
\end{aligned}
$$

As we have seen if $q / p<a<1$ the first integral is finite and clearly so is the second integral. The result now follows from Theorem 2.3.

3. The discrete case. We now consider the discrete analogue of Theorems 2.1 and 2.2 .

THEOREM 3.1. Let $\{K(m, n)\}$ be a nonnegative double sequence defined in $D=$ $\{(m, n) \in \mathbf{Z} \times \mathbf{Z} ; n \leqslant m\}$ such that $K(m, n)$ is nondecreasing in $n$. If $1 \leqslant q<p<$ $\infty$ and $\left\{U_{n}\right\},\left\{V_{n}\right\}$ are nonnegative sequences such that

$$
B \equiv\left\{\sum_{m=-\infty}^{\infty}\left[\left(\sum_{n=m}^{\infty} K(n, m)^{q} U_{n}\right)^{1 / q}\left(\sum_{n=-\infty}^{m} V_{n}^{1-p^{\prime}}\right)^{1 / q^{\prime}}\right]^{r} V_{m}^{1-p^{\prime}}\right\}^{1 / r}<\infty,
$$

$1 / r=1 / q-1 / p$, then for all sequences $\left\{a_{n}\right\}$

$$
\left(\sum_{n=-\infty}^{\infty} U_{n}\left|\sum_{k=-\infty}^{n} K(n, k) a_{k}\right|^{q}\right)^{1 / q} \leqslant B C\left(\sum_{n=-\infty}^{\infty}\left|a_{n}\right|^{p} V_{n}\right)^{1 / p}
$$

where $C=q^{1 / q}\left(p^{\prime}\right)^{1 / q^{\prime}}$.

Moreover, if $q=1,(3.2)$ implies (3.1).

Proof. Without loss of generality assume $a_{n} \geqslant 0$. Let $\sigma_{n}=V_{n}^{1-p^{\prime}}$; then using the elementary inequality

$$
\left(\sum_{k=-\infty}^{n} K(n, k) \sigma_{k} a_{k}\right)^{q} \leqslant q \sum_{k=-\infty}^{n} K(n, k) \sigma_{k} a_{k}\left(\sum_{l=-\infty}^{k} K(n, l) \sigma_{l} a_{l}\right)^{q-1}
$$

we obtain as in the proof of Theorem 2.1 via an interchange of summation Hölder's inequality and [1, Theorem 4.1]

$$
\sum_{n=-\infty}^{\infty} U_{n}\left(\sum_{k=-\infty}^{n} K(n, k) \sigma_{k} a_{k}\right)^{q} \leqslant B C\left(\sum_{n=-\infty}^{\infty} \sigma_{n} a_{n}^{p}\right)^{1 / p} .
$$

But this is equivalent to (3.2).

The case $q=1$ is as in Theorem 2.1 and we omit the details.

TheOREM 3.2. Let $\{K(m, n)\}$ be a nonnegative double sequence defined in $D=$ $\{(m, n) \in \mathbf{Z} \times \mathbf{Z} ; n \leqslant m\}$ such that $K(m, n)$ is nonincreasing in $m$. If $1 \leqslant q<p<$ $\infty$ and $\left\{U_{n}\right\},\left\{V_{n}\right\}$ nonnegative sequences satisfying

$$
B^{*} \equiv\left\{\sum_{m=-\infty}^{\infty}\left[\left(\sum_{n=-\infty}^{m} K(n, m)^{q} U_{n}\right)^{1 / q}\left(\sum_{n=m}^{\infty} V_{n}^{1-p^{\prime}}\right)^{1 / q^{\prime}}\right]^{r} V_{m}^{1-p^{\prime}}\right\}^{1 / r}<\infty
$$


$1 / r=1 / q-1 / p$, then for all sequences $\left\{a_{n}\right\}$

$$
\left(\sum_{n=-\infty}^{\infty} U_{n}\left|\sum_{k=n}^{\infty} K(k, n) a_{k}\right|^{q}\right)^{1 / q} \leqslant B^{*} C\left(\sum_{n=-\infty}^{\infty} V_{n}\left|a_{n}\right|^{p}\right)^{1 / p},
$$

where $C=q^{1 / q}\left(p^{\prime}\right)^{1 / q^{\prime}}$. Moreover, if $q=1$, (3.4) implies (3.2).

The proof of Theorem 3.2 is similar to that of Theorem 3.1 and therefore omitted.

We note that these results generalize certain inequalities of Leindler [2].

\section{REFERENCES}

1. K. F. Andersen and H. P. Heinig, Weighted norm inequalities for certain integral operators, SIAM J. Math. Anal. 14 (4) (1983), 834-844.

2. L. Leindler, Generalization of inequalities of Hardy and Littlewood, Acta Sci. Math. 31 (1970), 279-285.

3. V. I. Levin and S. B. Stečkin, Inequalities, Amer. Math. Soc. Transl. (2) 14 (1960), 1-29.

4. Wladimir Mazja, Einbettungssätze für Sobolevsche Räume, Teil 1, Teubner Texte zur Math., Teubner Verlag, Leipzig, 1979.

5. E. T. Sawyer, Weighted Lebesgue and Lorentz norm inequalities for the Hardy operator, Trans. Amer. Math. Soc. 281 (1) (1984), 329-337.

Department of Mathematical Sciences, McMaster University, Hamilton, Ontario L8S 4K1, CANADA 